\title{
Políticas de traducción de las Metamorfosis. Ovidio en la Inglaterra en el siglo XVIII
}

\author{
Ana Elena GonZÁLEZ TREviÑo \\ Universidad Nacional Autónoma de México
}

\begin{abstract}
Aun cuando la fama que alcanzó Ovidio en el Renacimiento ya estaba declinando, en el siglo XVIII hubo un resurgimiento del interés por traducir a los clásicos. Las traducciones resultantes dialogan de manera singular con valores ilustrados tales como la precisión filológica y el paradigma racionalista. Tal es el caso de la edición de las Metamorfosis publicada en 1717 en Inglaterra por el célebre librero Jacob Tonson, coordinada por Samuel Garth, en la que participaron dieciocho traductores notables entre los que se cuentan dos de los poetas más destacados del periodo, John Dryden y Alexander Pope. Médico de profesión pero gran amante de las letras, Garth estipula sus criterios y objetivos en la introducción al volumen. Dichos criterios fueron tachados de peculiares e idiosincráticos por sus contemporáneos, pero aun así, constituyen un documento muy significativo para la historia y la teoría de la traducción, así como de la recepción de Ovidio en esa época.
\end{abstract}

Palabras Clave: Ovidio; Metamorfosis; Jacob Tonson; Samuel Garth; John Dryden.

Even though the fame Ovid achieved in the Renaissance had already declined, the eighteenth century saw renewed interest in translating the classics. Resulting translations resonate particularly with Enlightenment values, such as philological precision and the rationalist paradigm. Such is the case of the 1717 edition of Ovid's Metamorphoses, published in England by Jacob Tonson and coordinated by Samuel Garth, with the collaboration of eighteen distinguished translators, including some of the most celebrated poets of the period: John Dryden and Alexander Pope. A trained physician who loved poetry, Garth explains his goals and criteria in the prologue to the work. Such criteria were discarded as peculiar and idiosyncratic by his contemporaries, but even if that were the case, they constitute a significant document for the history and theory of translation, and for the study of the reception of Ovid at the time.

KeYwords: Ovid; Metamorphoses; Jacob Tonson; Samuel Garth; John Dryden.

El librero Jacob Tonson, el Viejo (1656-1736), publicó en Londres el volumen que marcaría el final de su brillante carrera el 4 de julio de 1717: las Metamorfosis de Ovidio en quince libros, traducidos por las manos más eminentes [Ovid's Metamorphoses in 
Fifteen Books, Translated by the Most Eminent Hands] ${ }^{1}$ (Hopkins, 1988: 64). El Ovidio de Tonson es un elegante folio que contiene una traducción completa de las Metamorfosis en verso. El metro utilizado fue el pareado heroico, que consta de dísticos en pentámetro yámbico y que era el verso de moda en Inglaterra en aquel entonces. En la traducción participaron dieciocho traductores, entre los que se contaban las plumas más famosas de la época. John Dryden (1631-1700), poeta laureado y primer autor publicado por Tonson, después de morir le había dejado algunos fragmentos traducidos, y se dice que este hecho fue la principal motivación para el proyecto. ${ }^{2} \mathrm{Al}$ parecer, pocos años antes Dryden había contemplado la posibilidad de retraducir y modernizar las Metamorfosis que no se habían vuelto a traducir al inglés desde principios del siglo XVII (Hopkins, 1988: 67-68). Además de las traducciones de Dryden (que abarcaban, entre otras cosas, el Libro I; la historia de Ifis y Yante; la de Acis, Polifemo y Galatea; la de Meleagro y Atalanta, y la de Pigmalión y la estatua, y que en total comprenden más o menos la tercera parte del volumen), Jacob Tonson había heredado traducciones parciales de varios poetas más, como Nahum Tate (1652-1715) y Arthur Maynwaring (1668-1712), lo cual facilitó sin duda la tarea. Como se verá, este libro constituye un ejemplo señalado de proyecto editorial con metas comerciales bastante claras. En él se plasman los valores ilustrados que incluyen un nuevo modo de relacionarse con la Antigüedad clásica y con los compradores de libros de la época.

Por iniciativa de Tonson, en 1715 se hizo una convocatoria a todos los poetas y traductores que pudieran interesarse en participar. A Dryden se sumaron los célebres autores Alexander Pope, Joseph Addison, William Congreve y John Gay, entre otros. ${ }^{3}$ A cargo de la edición y coordinación estaba Samuel Garth, distinguido médico real y además poeta, a quien le fue encomendada esta labor en parte por pertenecer al Kit Cat Club, un círculo político y literario muy activo de tendencia liberal (Whig) fundado justamente por el librero Tonson, y al cual también pertenecían varios de los autores arriba citados. ${ }^{4}$ El propio Garth tradujo el libro XIV y fragmentos del XV de las $\mathrm{Me}$ tamorfosis, además de introducir la obra con un extenso y polémico prefacio. El volumen estaba dedicado a Carolina, princesa de Gales, cuyo retrato, pintado por Godfrey

\footnotetext{
${ }^{1}$ No debe confundirse con su sobrino, también llamado Jacob Tonson (a veces apodado el Joven), quien siguió los pasos de su tío como editor, aunque su fama no fue tan grande.

${ }^{2}$ En 1712 Tonson había publicado ya El arte de amar, con traducciones de Dryden y William Congreve (1670-1729).

${ }^{3}$ Pope, 1688-1744; Addison, 1672-1719; Congreve 1670-1729; Gay 1680-1732; Garth 1661-1719.

${ }^{4}$ David Hopkins enlista los siguientes: Joseph Addison, Alexander Catcott, William Congreve, Samuel Croxall, John Dryden, Laurence Eusden, Samuel Garth, John Gay, Stephen Harvey, Arthur Maynwaring, John Ozell, Alexander Pope, Nicholas Rose, Temple Stanyan, William Stonestreet, Nahum Tate, Thomas Vernon y Leonard Welsted. David Hopkins, "Dryden and the Garth-Tonson Metamorphoses", Review of English Studies, New Series, vol. XxxIX, núm. 183 (oup, 1988). Existía en Inglaterra una importante tradición de médicos literatos como Oliver Goldsmith, Tobías Smollett, Erasmus Darwin y John Arbuthnot que, así como Samuel Garth, también formaban parte de estos círculos. El propio Tonson era hijo de un cirujano.
} 
Kneller, retratista oficial del Kit Cat Club, figuraba en el frontispicio. ${ }^{5}$ Además, cada uno de los quince libros en que estaba dividido, iba precedido por un grabado alusivo a las historias ovidianas, al pie del cual aparecía una dedicatoria en letra cursiva para alguna dama eminente, casi siempre perteneciente a la nobleza, cuyo título figuraba acompañado de su escudo de armas. ${ }^{6}$

Muy distinguidos habían sido los traductores de Ovidio al inglés en siglos anteriores, empezando por William Caxton, cuya traducción completa de las Metamorfosis se publicó en 1480. (Chaucer y Gower solamente habían hecho traducciones parciales.) Lo que hizo Caxton en realidad no fue una traducción estrictamente hablando, sino una prosificación de una versión francesa muy difundida en el siglo XV que llevaba el título de Ovide Moralisé (Ovidio moralizado). Medio siglo después, Arthur Golding realizó una traducción muy importante publicada entre 1535 y 1537. Su importancia radica, entre otros méritos, en que fue la que consultó Shakespeare, a quien algunos llamaban "el Ovidio inglés". Casi un siglo después, entre 1621 y 1626, George Sandys publicó una traducción en dísticos heroicos, lo cual elevó considerablemente el nivel del esfuerzo literario con respecto a las traducciones anteriores. Fue tan influyente que se reeditaría al menos siete veces en el siglo XVII y principios del XVIII. Es la traducción que usó otro de los grandes poetas ingleses muy influidos por Ovidio: John Milton.

La siguiente traducción significativa es la que ahora nos ocupa, dirigida por Samuel Garth y publicada en 1717. Esta versión, impresa en folio, en dos tomos, se seguiría publicando hasta el siglo XIX revestida de una gran autoridad. Resulta interesante, empero, que de manera paralela, el librero Edmund Curll publicara una edición rival, traducida por George Sewell y otros, casi todos escritores despreciados con el apelativo de hacks, es decir, escritores jornaleros o a destajo. La edición de Curll costaba nada más seis chelines, baratísima en comparación con la de Tonson, que era de lujo y costaba dos guineas (a veintiún chelines por guinea) (Hopkins, 1988: 65).

El hecho de que existieran dos traducciones simultáneas nos revela que el contexto histórico era más que propicio para las reflexiones estéticas y sociales acerca de la naturaleza de la impermanencia, pues ambas ediciones nacen en una cultura en donde el cambio y las transformaciones de la identidad estaban a la orden del día, en gran parte por el nuevo estatus del reino (Gran Bretaña como tal se acababa de proclamar en 1707) y también por la gran volatilidad social y mercantil que se vivió en aquel periodo. Por mencionar sólo un ejemplo, Samuel Garth se lamenta en su prefacio diciendo que los únicos talentos que se valoran en ese momento son los del corredor de

${ }^{5}$ Se trata de Carolina de Ansbach, de la Casa Hohenzollen, quien se casó con Jorge II de Gran Bretaña en 1705. Ambos fueron muy cercanos a Robert Walpole (1676-1745), representante de la oposición Whig, y primero en ocupar el puesto de primer ministro de esa nación.

${ }^{6}$ Los artistas grabadores fueron Louis du Guernier, Michale van der Gucht, Elisha Kirkall y R. Smith (Hopkins, 1988: 64). Además de la Princesa de Gales, entre las damas aludidas se incluye a las duquesas de Kingston, Roxburghe, Newcastle, Rutland y St. Alban's; las condesas de Warwick, Lincoln y Burlington; las vizcondesas de Scudamore y Joronshend; la señora Walpole, esposa del primer ministro, y la princesa Ana, hija de los príncipes de Gales. 
la bolsa de valores, y que la gente prefiere leer los informes de las tasas de interés que cualquier otra cosa, por trascendente que ésta sea. ${ }^{7}$

Aunque la enorme popularidad de las Metamorfosis en el Renacimiento nunca volvería a ser igual, en los albores del siglo XVIII hubo un incuestionable resurgimiento de interés debido a las circunstancias históricas, económicas y sociales a las que acabo de aludir. El texto de Ovidio también se convirtió en un locus culturalmente favorable para la representación de la mutabilidad en otras áreas, además de la económica, por ejemplo la de la moda, e incluso se hicieron representaciones de los relatos ovidianos en botones decorativos y broches, así como en bordados que adornaban abrigos y vestidos (Laycock, 1997: 137). Los caprichos de la moda empalmaban admirablemente con las transformaciones radicales descritas por Ovidio. Lady Mary Wortley Montagu (1689-1762), escritora y viajera, reflexiona sobre la moda y sobre la nueva economía basada en el crédito bajo el gobierno liberal de los Whigs. En sus epístolas habla de la misteriosa 'metamorfosis' que han sufrido varias amigas suyas en aras de la moda, transformaciones tan radicales que parecen, dice, salidas de Ovidio. En otra carta describe su sensación de asombro al asistir a un baile de máscaras en Venecia cuando se vio a sí misma transformada, como si fuera un personaje de Ovidio (Laycock, 1997:151). Es en parte por eso que las Metamorfosis también se concibieron como un producto comercial dirigido a las mujeres, y con gran éxito. Las mujeres fueron grandes lectoras de las Metamorfosis (OakleyBrown, 2006: 123).

De hecho, en la segunda mitad del XVIII hubo una versión de la obra hecha por Abraham Fraunce que se hizo pensando exclusivamente en las mujeres lectoras, pues el título anuncia: Las metamorfosis de Ovidio, resumidas en un estilo poético inglés para uso y entretenimiento de las damas de la Gran Bretaña (1760) [Ovid's Metamorphoses Epitomized in an English Poetical Style, for the Use and Entertainment of the Ladies of Great Britain] (Oakley-Brown, 2006: 124). El prologuista escribe una dedicatoria a Lady Lennox, ${ }^{8}$ y explica que este epítome o resumen se hizo expresamente para el uso personal de dicha dama; enseguida extiende su discurso a todas las lectoras que por algún motivo no pudieran leer ni el original ni la totalidad de la obra en traducción. Este resumen, reconoce el prologuista, es un remedio, no una meta ideal. Sin embargo, aprovecha para explayarse acerca de las ventajas que tendría la práctica de educar a las mujeres del mismo modo que a los hombres, pues de ellas depende la prosperidad del reino. Aunque la traducción resumida está en prosa, la "expresión" está tomada (o incluso en parte plagiada) de Dryden, Addison, Pope y "los mejores traductores" (Ovidio, 1760: 2).

\footnotetext{
7 "The only Talents in Esteem at present are those of Exchange-All[e]y; one Tally is worth a Grove of Bays; and tis of much more Consequence to be well read in the Tables of Interest, and the Rise and Fall of the Stocks, than in the Revolution of Empires" (Garth, 1717: 20).

${ }^{8}$ Probablemente una de las célebres hijas de Charles Lennox, duque de Buckingham (1701-1750), aunque el texto no aclara cuál de ellas.
} 
El diseño del Ovidio de Garth y Tonson nos revela también que concebían al público femenino como consumidor ideal de las Metamorfosis en parte debido al papel cambiante de las mujeres en la sociedad, sobre todo en lo que se refiere a las relaciones entre los sexos. Los textos de Ovidio en el pasado se habían considerado lascivos e inmorales, y ciertamente no aptos para mujeres. Aunque cada vez más niñas habían tenido acceso a una educación humanista que incluía el estudio de los clásicos, con frecuencia se recomendaba que no leyeran este tipo de textos. El mundo ovidiano estaba poblado por seres que se dejaban arrastrar por las pasiones y sobre todo el deseo sexual, un contenido por demás subversivo en el contexto del siglo XVIII. Los dioses, quienes debieran ser modelos de conducta, eran injustos e irascibles violadores, volubles en sus designios, parciales al otorgar sus favores, ignorantes de la verdad, groseros en su expresión, brutales contra sus enemigos o contra su propia familia (Garth, 1717: xv-xvi). A pesar de todo, explica Garth, las Metamorfosis contienen excelentes lecciones morales que procede a enumerar, $\mathrm{y}$ entre las que se cuentan las siguientes: que los padres no deben consentirle todo a sus hijos (Faetonte); que el exceso de devoción, lo mismo que poner a prueba a los dioses, produce resultados desastrosos (Licaon y Penteo); que vender nuestra patria es una infamia (Minos y Escila); que la gloria se alcanza tras mucho sufrimiento (Heracles); que nada es seguro, pero que no debemos desesperar (Ariadna), y muchas más (Garth, 1717: xvii).

El hecho de publicar la obra intercalando nombres de damas respetables de la sociedad replanteaba la participación de la mujer en la cultura libresca e incluso política del momento. Aunque la mujer se hubiera concebido más como consumidora que como productora (traductora, recreadora) de las Metamorfosis (Cotterill, 2004: passim; Oakley-Brown, 2006: 123), es indudable que estamos presenciando un cambio histórico gradual hacia la liberalización de las costumbres. En su dedicatoria a la princesa Carolina, Garth se preocupa por exaltar las virtudes de esta dama a pesar de reconocer que se vive una era de "depravación moral". Todos los refinamientos de Carolina, en parte derivados de su noble cuna, en parte de su virtud innata, servirán para trasladar la obra de Ovidio del exilio cultural a la "Fortaleza de la Libertad", de la barbarie a la cortesía (Garth, 1717: sig. Ar, A2r). Enmarcar las fábulas con los nombres de las damas inscribe a la mujer como garante de la lectura inteligente incluso de las historias más depravadas, al tiempo que revela a un público femenino que, a pesar de estar familiarizado con las historias, no por ello pierde su virtud.

En el ámbito de la crítica erudita, con frecuencia se había representado a Ovidio como un poeta inferior a Virgilio, por lo cual no sólo se requería una justificación ética, sino también estética que lo redimiera. Con la objetividad característica de la argumentación ilustrada, Garth estudia las debilidades y los aciertos poéticos de Ovidio, y en numerosas ocasiones compara pasajes específicos de éste con Virgilio para explicar que no desmerece. Todas las citas están en latín, lo cual facilitaba que los lectores pudieran memorizar algún pasaje en la lengua original para recitarlo en público cuando la ocasión lo ameritara. Por otra parte, Garth sostiene que el espíritu 
excesivamente crítico de los ingleses ha sido injusto con Ovidio, y espera que su estudio sirva para revalorarlo, aun cuando siempre siga a la sombra del gran Virgilio. ${ }^{9}$

A principios del siglo XVIII, las traducciones colectivas gozaron de una popularidad considerable. Las epístolas y el Arte de amar de Ovidio, así como las Vidas de Plutarco se publicaron por este método. La razón que se arguye para justificar la frecuencia con que se utilizó este recurso es de índole económica, pues la gran demanda que tenía este tipo de obra no se podía satisfacer de otra manera a corto plazo. Las traducciones contribuyeron a impulsar a la industria editorial, pues contribuían de manera importante a la educación de la burguesía que no hubiera recibido los beneficios de la educación formal, pues hasta entonces había sido un privilegio casi exclusivo de la aristocracia (Oakley-Brown, 2006: 95). Los clásicos traducidos por escritores célebres no sólo se volvían accesibles gracias a la proliferación de ediciones, sino que también servían para reforzar la nueva posición de ascendencia social de las clases comerciales. En otras palabras, la lectura de los clásicos era una modalidad de capital cultural cuyo conocimiento y posesión llegó a constituir una divisa social considerable.

Los participantes en el proyecto de las Metamorfosis de Tonson trabajaron a lo largo de un periodo de treinta años, si bien no de manera sistemática - tomando en cuenta las traducciones realizadas años antes por traductores ya fallecidos-, lo que, aunado a las diversas técnicas adoptadas por cada uno de los dieciocho traductores, produjo un volumen carente de unidad y coherencia estilística. En principio, todos seguirían el método de Dryden, cuyo objetivo general era simplemente hacer una paráfrasis en pareados heroicos, sin ser ni una traducción precisa, ni una imitación del todo libre, aunque las versiones de Dryden son sustancialmente más extensas que el original (Hooley, 2014: 348). Garth no busca disimular esta diversidad; al contrario, la presenta como una especie de virtud. Argumenta que debido a que los lectores no son "invitados formales", sino que llegan al texto de manera accidental, se deben contentar con lo que encuentren en el espectáculo ovidiano sin mayor exigencia (Garth, 1717: ii). ${ }^{10}$ Argumenta que la falta de unidad en realidad no afecta al proyecto de manera negativa, aunado al hecho de que el propósito del librero Tonson se limitaba a satisfacer una demanda comercial por parte de un público ávido de tener las Metamorfosis "completas" y dispuesto a pagar por una edición de lujo. Así, Tonson consigue una obra ideal para el consumo, tanto para la tertulia literaria como para el salón de clases (de hecho, con el tiempo el estudio de Ovidio se relegaría más y más al ámbito académico). Tonson logró exprimir la fama póstuma de Dryden un poco más y Garth consiguió posicionarse como su sucesor literario, al menos momentáneamente.

El prefacio que escribió fue muy criticado por algunos en las décadas siguientes. Joseph Warton, en 1756, afirma que las opiniones de Garth son bastante "extrañas", y

9 "[...] I ought to be on this occasion an Advocate for Ovid, whom I think is too much run down at present by the critical Spirit of this Nation..." (Garth, 1717: xv).

10 "Since therefore the Readers are not solemnly invited to an Entertainment, but come to it accidentally; they ought to be contented with what they find" (Garth, 1717: ii). 
Samuel Johnson, hacia 1781, sostiene que su introducción está escrita más con ostentación que con destreza, que sus ideas están a medio formar y que sus materiales son confusos (Oakley-Brown, 2006: 96; Johnson, 1807 [1781]: 9). La crítica más severa es la que describe esta traducción como un triunfo comercial en detrimento del impulso literario genuino (Oakley-Brown, 2006: 96). A pesar de todo, el proyecto editorial de Garth sigue siendo un objeto de gran interés en cuanto a la política cultural de la traducción en la Inglaterra del siglo XVIII.

En este periodo, en consonancia con los principios de la Real Sociedad (la Royal Society), se buscaba lograr un lenguaje en donde imperara la razón, en donde la palabra fuera el equivalente más exacto posible del objeto que designaba, y en donde la imaginación tenía muy poco que decir. Además, existía una gran presión por regularizar y estabilizar la lengua inglesa, de manera particular en el mundo editorial, como una instancia más del deseo prevaleciente de establecer el orden en todos los ámbitos. Las Metamorfosis eran una obra que se prestaba mucho para este proyecto cultural en la medida en que en ellas se tocaban temas referentes a una multiplicidad de disciplinas, entre las que encontramos la astronomía, el estilo, la política, el amor, la retórica y las ciencias en general.

Más aún, a partir de 1660, año de la restauración de la monarquía en Inglaterra, se extendió una actitud de escepticismo hacia la interpretación alegórica de los mitos, sobre todo porque se habían leído siempre a la luz del cristianismo. Esto posibilitó nuevas maneras de acercarse a Ovidio, por ejemplo, estableciendo relaciones alusivas entre el texto y los acontecimientos del momento (Horowitz, 1988: 356). Así pues, Garth explica en su prefacio que las "alegorías deben ser obvias y no como meteoros en el aire, que representan una figura distinta para cada observador" ["Allegories should be obvious, and not like Meteors in the Air, which represent a different Figure to every Eye", en Oakley-Brown, 2006: 99]. Esta postura difiere considerablemente del espíritu traductológico de épocas anteriores. Ya en el siglo XVIII, Garth lleva al extremo la necesidad de precisión y acaba, por ejemplo, por encontrar significado personal en algunas de las anécdotas del texto. Siendo médico, le llama particularmente la atención un pasaje del libro XV en el que se relata el viaje de Esculapio a Roma en forma de serpiente. Él lo interpreta como un modelo para la profesión médica en el sentido de que el animal elegido por Esculapio no tiene manos para indicar que los médicos no deben cobrar por sus servicios, aunque los pacientes deben recompensarlos en señal de agradecimiento. Y de hecho Garth, que era miembro del Colegio Real de Médicos [Royal College of Physicians], veinte años antes había sido uno de los principales promotores de la fundación de un dispensario en donde se diera atención médica gratuita y medicamentos a precio de descuento (Oakley-Brown, 2006: 99; Garth, 1717: xviii). Otros pasajes de su prefacio se dedican a vincular episodios de las Metamorfosis con los acontecimientos de su momento histórico. En su poema Claremont, inspirado en Ovidio, Garth relaciona el relato de Narciso y Eco con la historia de la sucesión hanoveriana en Inglaterra y la nueva identidad británica del recién fundado imperio (Oakley-Brown, 2006: 103).

También es significativo que, a la muerte del antiguo poeta laureado, John Dryden, quien fuera el primero de este grupo en emprender la traducción de las Metamorfosis, 
varios designaran sucesor suyo precisamente a Samuel Garth (Oakley-Brown, 2006: 100). De hecho, hay quien establece una filiación directa que va de Ovidio a Dryden y de Dryden a Garth, lo cual da al proyecto de traducción una importancia cultural que va más allá de la traducción misma, aun cuando el nombre de Garth se conozca poco en nuestros días.

Por otra parte, Garth sabía que sus Metamorfosis planteaban un desafío a la traducción de George Sandys, publicada casi un siglo antes en 1632 con el título de Metamorphoses Englished. La traducción de Sandys se publicó a todo lo largo del siglo XVII, e incluso hasta principios del XVIII. Liz Oakley-Brown sostiene que la traducción de George Sandys se había hecho pensando en exaltar la figura del entonces monarca, Carlos I, primer y último monarca absoluto de Inglaterra, mientras que la edición de Garth estaba destinada a exaltar los valores del partido liberal de los Whigs, y los intereses parlamentarios. En otras palabras, son traducciones que defienden posturas políticas opuestas. Sin embargo, la crítica que le hace Garth a su antecesor no se refiere más que a cuestiones estrictamente traductológicas. Acusa a Sandys de haber hecho una traducción literal, misma que el texto ovidiano no resiste. En parte esto se debe a que el mismo Ovidio dejó la obra inacabada y sin revisar, por lo que quedaron muchos pasajes que, según Garth, en una revisión habrían sido eliminados. El defecto principal de Sandys es que no se atrevió a intervenir editorialmente en el texto debido a una veneración excesiva del original derivada de una timidez crítica propia del momento histórico en que Sandys tradujo. En la actualidad, explica Garth, es decir, en la modernidad del siglo XVIII, el traductor debe procurar "cuidar la gracia del poema y esconder sus imperfecciones; copiar sus bellezas, y ocultar sus fallas" (en Oakley-Brown, 2006: 106).

Esta actitud editorial tan activa parte de un supuesto cultural sumamente provocador mediante el cual el traductor/editor se pregunta cómo habría sido la obra de haberse escrito originalmente en su propio contexto histórico. El modelo y la justificación para este enfoque, por supuesto, es Dryden, quien se dice logró domesticar a Ovidio por completo, y lo hizo sonar como si fuera exactamente un caballero inglés de fines del siglo XVII (a diferencia de Sandys, que había preservado la sintaxis latina tanto como lo permite la lengua inglesa) (Hooley, 2014: 348). Sin dejar de lado el texto original, éste serviría más para consulta que como fuente primaria jerárquicamente superior a la versión actualizada, aun cuando mucha más gente se acercaría a esta última.

La traducción de las Metamorfosis de Sandys había fungido como una especie de traducción oficial, citada por diversos autores desde mediados del siglo XVII hasta finales del siglo XVIII. Este hecho le pesó considerablemente a Garth y también al célebre poeta Alexander Pope, que escribió una balada satírica sobre Sandys titulada "El fantasma de Sandys: o, una balada realmente nueva sobre las nuevas Metamorfosis de Ovidio" [Sandys Ghost: Or, A Proper New Ballad on the New Ovid's 'Metamorphosis'], en la que se sugiere que el fantasma de Sandys acecha a todos los traductores ingleses que le siguieron (Oakley-Brown, 2006: 108). En su prefacio, Garth trata de deslindarse de Sandys y sus métodos de traducción, pero, entre todos los traductores que participaron en el proyecto, paradójicamente resulta ser el que más fielmente lo sigue. 
En 1759 se publicó Una nueva traducción de las Metamorfosis de Ovidio en pro$s a . .$. , edición bilingüe dirigida a un público escolar masculino [A New Translation of Ovid's Metamorphoses into English Prose, as near the original as the different Idioms of the Latin and English languages will allow... for the use of schools as well as of Private Gentlemen]. No se da ningún crédito de la traducción ni de las notas, pero en el frontispicio se incluye una lista de trece libreros que encargaron el proyecto, lo que revela probablemente un público escolar mucho más amplio, del tipo que se podría esperar hoy en día para un libro de texto. Cabe observar también que el prefacio claramente toma sin empacho pasajes enteros del prefacio de Garth, sin dar ninguna atribución. La obra resulta de interés además por la problemática tipográfica que supuso incluir en cada página el texto latino, la traducción al inglés y cuantiosas notas explicativas de crítica textual. Sobra decir que esta edición no tiene ni dedicatoria ni ilustraciones, pues se pensó para uso académico, y no social.

Las transformaciones ovidianas se dieron también en el ámbito de la novela. Autores como Eliza Haywood, Samuel Richardson y Tobías Smollett con frecuencia aluden a las Metamorfosis para describir los momentos en los que sus personajes adoptan algún disfraz, especialmente un disfraz que haga parecer que pertenecen a otra clase social. En todos los casos, se observa una tendencia a domesticar el texto ovidiano para volverlo más afín al contexto inmediato. Así, el público que no estuviera familiarizado con Ovidio conocería un poco de este autor a través de sus versiones noveladas, y el público más instruido podría reconocer el pedigrí clásico de las nuevas formas literarias (Horowitz, 2014: 362). También se podría argumentar que las historias ovidianas tienen mucho de melodrama, lo cual las vuelve compatibles con el género novelesco. Resultaría interesante investigar este asunto desde la perspectiva de la recepción de ambos por parte del público femenino. Aunque rebasa nuestro presente objetivo, cabe señalar que los temas del rapto y la violación se han tratado con frecuencia, sobre todo a la luz del papel cambiante de la mujer en la sociedad. A pesar de que tuvieron que pasar muchos siglos antes de que se considerara que la violación es un crimen, se ha sugerido que Ovidio es el primero en retratar el sufrimiento femenino ante la persecución, así como el trauma de la violación que deja a la mujer "cambiada", metamorfoseada, o bien en la cual el hombre se transforma en bestia o en alguna entidad irracional para cometer ese acto tan inhumano (Bloch, 2014: passim; Cruikshank, 2010: 5, 306, 311).

Para concluir, simplemente reitero que, a pesar de todo, la influencia de las Metamorfosis de Garth y compañía en este periodo es considerable, aunque el reconocimiento que se les dio no deja de ser ambivalente.

\section{Obras citadas}

BLoch, Nikki. 2014. "Patterns of Rape in Ovid's Metamorphoses". Tesis no publicada. Boulder: Universidad de Colorado. 
CotTerill, Anne. 2004. "Dryden's Fables and the Judgment of Art", en Steven N. Zwicker, ed., The Cambridge Companion to John Dryden. Cambridge, N. Y.: Cambridge University Press. Pp. 259-279.

Cruikshank, Dan. 2010. London's Sinful Secret: The Bawdy History and Very Public Passions of London's Georgian Age. Londres: Saint Martin's Press.

GARTH, Samuel, ed. 1717. Ovid's Metamorphoses Translated by the Most Eminent Hands. Adorn'd with Sculptures. Londres: Jacob Tonson. ECCO Documento Gale CW111474903.

Hooley, Dan. 2014. "Ovid Translated: Early Modern Versions of the Metamorphoses", en John F. Miller y Carol E. Newlands, eds., A Handbook to the Reception of Ovid. Hoboken, N. J.: John Wiley \& Sons.

HopkIns, David. 1988. "Dryden and the Garth-Tonson Metamorphoses". Review of English Studies, New Series, vol. XxxIX, núm. 183. Oxford: Oxford University Press. Pp. 64-74.

Horowitz, James M. 2014. "Ovid in Restoration and Eighteenth-Century England", en John F. Miller y Carol E. NewlandS, eds., A Handbook to the Reception of Ovid. Hoboken, N. J.: John Wiley \& Sons.

Johnson, Samuel. 1807 [1781]. The Poetical Works of Sir Samuel Garth, with a Life of the Author. Londres: Samuel Bagster.

LAYCOCK, Deborah. 1997. "Shapeshifting: Fashion, Gender and Metamorphosis in Eighteenth-Century England", en Lori Hope LefKoviTZ, ed., Textual Bodies: Changing Boundaries of Literary Representation. Nueva York: SUNY.

OAKLEY-Brown, Liz. 2006. "In Arachne's Trace: Women as Translators of the Metamorphoses". Ovid and the Cultural Politics of Translation in Early Modern England. Farnham, Surrey: Ashgate.

Ovidio. 1760. Ovid's Metamorphosis Epitomized in an English Poetical Style, for the Use and Entertainment of the Ladies of Great Britain. Londres: Robert Horsfield. 